\title{
DE LA FALTA DE NORMA EXPRESA Y ESPECIAL QUE REGULE LA INTERRUPCIÓN CIVIL DE LA PRESCRIPCIÓN ESPECIAL DE CORTO TIEMPO EN MATERIA DIVERSA A LA PATRIMONIAL, ESPECÍFICAMENTE EN EL SUPUESTO DE PLURALIDAD DE SUJETOS PASIVOS. COMENTARIO A SENTENCIA DE LA CORTE de APELACIONES DE ANTOFAgASTA DE 10 DE MARZO DE 2011, ROL $N^{\circ}$ 10-2011
}

\section{AleXIS MondaCA MirandA*}

\section{1) INTRODUCCIÓN}

El reconocimiento, como todo acto y declaración de voluntad, puede verse contaminado por la presencia de un vicio que afecte la voluntad de su autor, ya sea el error, la fuerza o el dolo. Esta materia se encuentra regulada en el artículo 202 del Código Civil, norma que establece lo siguiente: "La acción para impetrar la nulidad del acto de reconocimiento por vicios de la voluntad prescribirá en el plazo de un año, contado desde la fecha de su otorgamiento o, en el caso de fuerza, desde el día en que ésta hubiera cesado". Podemos apreciar lo breve del plazo de prescripción de la acción de nulidad del reconocimiento por vicios del consentimiento ${ }^{1}$, especialmente si se le compara con el plazo de cuatro años que establece en materia de nulidad patrimonial el inciso $1^{\circ}$ del artículo 1691 del Código de Bello.

Considerando lo anterior, el peligro para aquel cuya voluntad se encuentra viciada radica en lo breve del término para deducir la correspondiente acción de nulidad. Esto resulta ser especialmente cierto a propósito del error y del dolo, puesto que, en tales casos, el plazo empieza a correr desde que se otorgó el reconocimiento y no desde el descubrimiento del yerro o del engaño, según corresponda; luego, es perfectamente posible que aquel que reconoce tenga conocimiento del vicio que afectó a su voluntad una vez transcurrido el término de un ańo. Súmese a lo anterior la siguiente consideración: el que reconoce carece de titularidad activa

Profesor de Derecho civil de la Escuela de Derecho de la Universidad Católica del Norte, Antofagasta. Becario Conicyt. Doctor en Derecho, Programa de Doctorado en Derecho de la Facultad de Derecho de la Pontificia Universidad Católica de Valparaíso.

1 El plazo de un año de prescripción es de los más breves del Código Civil, siendo superior solo al de pocos términos, tales como, el de seis meses establecido para la querella de restablecimiento (inc. $1^{\circ} \mathrm{del}$ art. 928), y el que puede pactarse a propósito del pacto comisorio calificado con relación a la obligación de pagar el precio en la compraventa, el cual prescribe en el plazo prefijado por las partes, siempre que no exceda de cuatro años (inc. $1^{\circ}$ del art. 1880). 
para efectos de impugnar la filiación establecida mediante reconocimiento $^{2}$, lo que es coherente con la característica de irrevocabilidad que posee dicha figura. En efecto, en poco segura posición quedaría el hijo si el autor del reconocimiento, unilateralmente, pudiese dejar este sin efecto, y en consecuencia, eliminar la determinación de la paternidad. Por lo tanto, de gran relevancia resulta ser la interposición de la acción de nulidad dentro de plazo legal por parte de quien ha sufrido el error, la fuerza o el dolo, de forma tal de interrumpir civilmente la prescripción. En caso contrario, se extinguirá su acción por prescripción extintiva.

El sujeto pasivo, por su parte, debe estar atento al transcurso del tiempo para una eventual interposición de una excepción de prescripción extintiva, y como eventual consecuencia de esto, el rechazo de la demanda en su contra deducida. Pero, si se decreta la nulidad de todo lo obrado, si además existe pluralidad de sujetos pasivos, y uno de ellos fue notificado de la demanda con anterioridad a la mencionada resolución, y solo con posterioridad a esta se notificó, esta vez a ambos, ¿qué sucede con la primera notificación de la demanda?, ¿̨ha provocado la interrupción de la acción de nulidad del reconocimiento? Pensemos en las consecuencias de lo anterior: si se entendiese que debiese quedar sin efecto la inicial notificación de la demanda, entonces, no se habría interrumpido la prescripción, y como el plazo de esta ha seguido corriendo, podría suceder que ya hubiese transcurrido íntegramente, con lo que el actor quedaría en una desfavorable posición, especialmente en los supuestos en que, efectivamente, haya incurrido en un error, o peor aún, sea la víctima de un dolo.

Esto es lo que se discute en el fallo que comentamos; los efectos que produce la declaración de la nulidad de todo lo obrado con relación a la interrupción del plazo de prescripción, en este caso, de la acción de nulidad del reconocimiento, si existe pluralidad de demandados notificados, uno en forma previa, y ambos con posterioridad a la aludida resolución judicial.

\section{2) RESUMEN DE LOS HECHOS}

En primera instancia, el juicio se inició por demanda de nulidad de reconocimiento efectuado por el actor a favor de su supuesto hijo, en vir-

En conformidad a lo dispuesto en el art. 216 en relación con el art. 214, ambos del C.C., son sujetos activos de la acción de impugnación de la paternidad determinada por reconocimiento: el propio reconocido por sí; o si fuese incapaz, su representante legal en interés de este; si muere desconociendo el reconocimiento o antes de vencido el término de que dispone para impugnar la paternidad, corresponderá la acción a sus herederos; finalmente, la acción corresponde a todo aquel que pruebe un interés actual en ello. Véase, Gómez de LA Torre Vargas, Maricruz. (2007). El sistema filiativo chileno. Santiago: Editorial Jurídica de Chile, p. 103. Además, consúltese, Ramos Pazos, René. (2005). Derecho de familia, t. II, 5 a edic., Santiago: Editorial Jurídica de Chile, pp. 419-420. 
tud del cual, se determinó la paternidad no matrimonial del demandante. Con posterioridad, según el relato del actor, se habría percatado de que su voluntad estaba viciada por dolo. En efecto, afirmó que la madre del menor fraguó un ardid tendiente a hacerle creer que era el verdadero padre del hijo objeto de su posterior reconocimiento. Encontrándose dentro de plazo legal, dedujo la acción de nulidad correspondiente.

Antes de que transcurriese el plazo de prescripción, el actor notificó legalmente la demanda a la madre, representante legal del menor. A mayor detalle, con fecha 16 de junio de 2009 fue interpuesta la acción, la que se notificó en forma debida, a la madre, el 23 de junio del mismo año, produciéndose, de esta manera, según lo expresa el tribunal de segunda instancia en el considerando octavo, la interrupción de la prescripción, en conformidad a lo prescrito en el $\mathrm{n}^{\circ} 2$ del artículo 2523 del Código Civil (norma relativa a la prescripción de corto tiempo propiamente tal). El problema que se produjo fue que la demanda no se notificó al hijo.

Con posterioridad, advirtiendo esta situación, el 29 de octubre del 2010, el sentenciador de primer grado decretó la nulidad de todo lo obrado. En virtud de lo último, se ordenó deducir y notificar la demanda al hijo, lo que fue cumplido el 11 de noviembre del mismo año, notificándose, finalmente, tanto a la madre como al hijo la acción de nulidad.

Sucedió que, intentando aprovecharse del escenario descrito, la madre opuso la excepción de prescripción extintiva, puesto que al producirse la nulidad de todo lo obrado, debía entenderse que no había operado la interrupción de la prescripción, razón por la cual, el plazo de prescripción habría continuado corriendo. Por lo tanto, al haber transcurrido en forma íntegra el plazo de prescripción establecido en el artículo 202 del Código Civil con anterioridad a la notificación del 11 de noviembre, correspondía que el tribunal a quo declarase prescrita la acción de nulidad del reconocimiento.

La sentencia de primera instancia del Tribunal de Familia de Antofagasta, de 4 de enero de 2011, acogió la excepción de prescripción opuesta por la demandada, rechazando, por lo tanto, la acción de nulidad de reconocimiento deducida por el actor fundada en el dolo de la madre del menor de edad que habría causado un error en el demandante al entender este que efectivamente era el padre del hijo de la demandada. En contra de esta resolución se dedujo recurso de apelación.

En segunda instancia, la situación cambió totalmente. En este sentido, la Ilustrísima Corte de Apelaciones de Antofagasta, en fallo de 10 de marzo de 2011 revoca la sentencia de primera instancia, desestimando, en consecuencia, la prescripción declarada por el juez a quo, ordenando que debía reanudarse la tramitación del proceso. 


\section{3) ANÁLISIS DE LA SENTENCIA}

\section{1.) LA PRESCRIPCIÓN DE LA ACCIÓN DE NULIDAD DE RECONOCI- MIENTO ES UNA PRESCRIPCIÓN "ESPECIAL" DE CORTO TIEMPO, Y COMO TAL, SE INTERRUMPE CIVILMENTE MEDIANTE ACCIÓN JUDICIAL, EN LOS TÉRMINOS DEL ARTÍCULO 2518 DEL CÓDIGO CIVIL EN RELACIÓN CON EL ARTÍCULO 2503 DEL MISMO CUERPO NORMATIVO}

Como lo hace presente el tribunal ad quem, en el considerando primero de su fallo, el objeto de la apelación fue "determinar si existió interrupción de la prescripción de la 'acción de nulidad del reconocimiento', o si por el contrario, transcurrió el plazo de prescripción de la acción que establece el artículo 202 del Código Civil respecto del menor”. Para una adecuado tratamiento de esta cuestión, debemos tener presente que se trata de una prescripción especial de corto tiempo, es decir, no de una prescripción de corto tiempo propiamente tal o "presuntiva de pago", como lo es la de tres, dos y un año, reguladas en los artículos 2521 a 2523 del Código Civil ${ }^{3}$. A las prescripciones especiales de corto tiempo le ha dedicado el Código de Bello una exigua regulación. En efecto, solo se disciplinan en el artículo 2524, en cuya virtud, "Las prescripciones de corto tiempo a que están sujetas las acciones especiales que nacen de ciertos actos o contratos, se mencionan en los titulos respectivos, y corren también contra toda persona; salvo que expresamente se establezca otra regla". Podemos apreciar que dentro de este breve tratamiento del artículo 2524 no se realiza referencia alguna a la interrupción de la prescripción; luego, deberá acudirse a la regla general del artículo 2518. Este aspecto no fue apreciado por el tribunal, el que califica la prescripción de la acción de nulidad de reconocimiento por vicios de la voluntad como una prescripción de corto

La distinción entre prescripciones de corto tiempo propiamente tales y prescripciones especiales de corto tiempo se funda en el tenor del art. 2523, el que establece reglas aplicables solo a las prescripciones de los dos artículos precedentes, y no para la de la norma posterior. Esta clasificación no es baladí, puesto que existen diversas consecuencias jurídicas resultantes de tratarse de una o de otra. Así, las prescripciones de corto tiempo propiamente tales poseen una reglamentación específica, y por lo tanto, diversa de la que corresponde a las prescripciones especiales de corto tiempo. En el mismo sentido, en conformidad a lo establecido en el inc. final del art. 2523, si opera la interrupción, sucede a las prescripciones de corto tiempo propiamente tales la del artículo 2515, situación que no ocurre a propósito de las prescripciones especiales de corto tiempo. Véase, Domínguez Águila, Ramón. (2004). La prescripción extintiva. Doctrina y jurisprudencia. Santiago: Editorial Jurídica de Chile, p. 335; Rodríguez Grez, Pablo. (2008). Extinción no convencional de las obligaciones, Vol. II, Santiago: Editorial Jurídica de Chile, p. 325; Troncoso Larronde, Hernán. (2006), De las obligaciones, 4a edic., Santiago: LexisNexis, p. 318; Vial del Río, Víctor. (2003). Manual del Derecho de las obligaciones en el Código Civil chileno. Santiago: Editorial Biblioteca Americana, pp. 326-327; y, Barcia Lehmann, Rodrigo. (2008). Lecciones de Derecho civil chileno, t. III, Santiago: Editorial Jurídica de Chile, p. 207. 
tiempo, sin percatarse que, específicamente, se trata de una prescripción "especial" de corto tiempo. Así, en el considerando segundo, se establece lo siguiente: "Este plazo de un año es un plazo especial de prescripción de corto tiempo. Que el artículo 2523 del mismo código citado que regula las prescripciones de corto tiempo expresa en su numeral $2^{\circ}$ que se interrumpe el plazo de prescripción 'desde que interviene requerimiento"”.

Con todo, esta inadvertencia no influye en la resolución del fallo, puesto que, tanto a propósito de la prescripción de largo tiempo como respecto de la de corto tiempo, sea esta propiamente tal o "especial", resulta claro que la notificación en regla de una acción o gestión del sujeto activo por la cual solicita la debida protección a su derecho interrumpe civilmente la prescripción. Y esto fue lo que sucedió en la especie. En este sentido, ninguna duda deja el texto del artículo 2518, referido a la prescripción de largo tiempo, al utilizar la expresión "demanda judicial"; en el caso de la prescripción de corto tiempo propiamente tal, prescribe el $\mathrm{n}^{\circ}$ 2 del artículo 2523, "Desde que interviene requerimiento", lo que se discute es si el requerimiento puede ser extrajudicial, pero no si este puede efectuarse en sede judicial ${ }^{4}$; y con respecto a la prescripción "especial" de corto tiempo, como ya hemos indicado, no existe regulación sobre el particular, de ahí que proceda aplicar la regla del artículo $2518^{5}$.

Debemos tener presente en este análisis que la prescripción extintiva, sea esta de largo o de corto tiempo, debe reunir copulativamente los requisitos que a continuación se indican: existencia de una acción prescriptible; el transcurso del término legal respectivo; y la inactividad de las partes ${ }^{6}$. En cuanto al primero, por cierto que en la especie se cumple, existe un expreso plazo de prescripción para la acción que originó la dictación de la sentencia que comentamos prescrito en el artículo 202 del Código Civil. El segundo y tercer requisito provocaron la discusión de las partes ¿̇transcurrió en forma íntegra el plazo de prescripción?, o, por el contrario, ¿este fue interrumpido civilmente por el actor, en conformidad a Derecho, poniendo fin a su inactividad?

La deducción de la demanda no interrumpe la prescripción en la medida que se dé alguno de los supuestos indicados en el artículo 2503 del Código Civil, esto es, que la demanda no se haya notificado en forma legal; que se declare el desistimiento de la acción o el abandono de la instancia; o que el demandado obtenga sentencia de absolución. En el

4 Véase, Alessandri R., Arturo, Somarriva U., Manuel y Vodanovic H., Antonio. (2004). Tratado de las obligaciones. Modificación y extinción de las obligaciones, Vol. III, $2^{a}$ edic., Santiago: Editorial Jurídica de Chile, pp. 208-209 y 225-227; y Meza Barros, Ramón. (1994). Manual de Derecho civil. De las obligaciones. Santiago: Editorial Jurídica de Chile, pp. 521-522.

5 Larraín Ríos, Hernán. (2003). Teoría general de las obligaciones. Santiago: LexisNexis, p. 457. También, véase, Domínguez ÁGuila (2004) 364.

6 Abeliuk Manasevich, René. (2003). Las obligaciones, t. II, 4a edic., Santiago: Editorial Jurídica de Chile, p. 1080. 
considerando decimocuarto, refiriéndose al mencionado artículo, señaló el tribunal ad quem que: "En el caso de autos no se da ninguna de estas figuras jurídicas, porque lo que efectivamente existió fue una nulidad de todo lo obrado, no por haberse hecho una notificación ilegal, sino por otro motivo: falta de legitimación pasiva respecto al hijo menor de edad". En efecto, razón tiene el sentenciador, los medios en virtud de los cuales la notificación de la demanda no interrumpe civilmente la prescripción están señalados de forma taxativa en el artículo 2503. Y en parte alguna se menciona a la nulidad de todo lo obrado. Un doble argumento de texto nos conduce a afirmar la taxatividad de la norma en comento: en primer lugar, el inciso $2^{\circ}$ del precepto establece que "Solo el que ha intentado este recurso podrá alegar la interrupción, y ni aún él en los casos siguientes". En segundo término, reforzada se ve esta conclusión al tenor del inciso final de la misma norma: "En estos tres casos se entenderá no haber sido interrumpida la prescripción de la demanda"7. Bastante claro nos parece el tenor literal del artículo.

En el caso que analizamos, en opinión del tribunal de alzada, al notificarse la demanda en el mes de junio, se interrumpió civilmente la prescripción. Justifica su decisión el sentenciador en el considerando segundo, al sostener que: "Incluso si aplicamos el artículo 2503 llegamos a la conclusión que la prescripción se interrumpió por recurso judicial”.

\section{2.) INEXISTENCIA DE UNA NORMA, EN EL DERECHO DE FAMILIA, QUE ESTABLEZCA SI LA INTERRUPCIÓN DE LA PRESCRIPCIÓN A FAVOR DE UNO DE LOS SUJETOS PASIVOS BENEFICIA TAMBIÉN A LOS OTROS}

El inciso $1^{\circ}$ del artículo 265 del Código Civil, analizando la representación legal de los hijos, específicamente en su aspecto judicial, dispone que en las acciones civiles contra el hijo deberá el actor dirigirse al padre o madre que tenga la patria potestad "para que autorice o represente al hijo en la litis". Por su parte, según lo establecido en el artículo 245 del mismo cuerpo texto legal, si los padres viven separados, la patria potestad será ejercida por aquel que tenga el cuidado personal del hijo en conformidad al artículo 225. En el juicio cuya sentencia comentamos, por tanto, corresponde el ejercicio de la patria potestad a la madre. Así se establece en el considerando tercero del fallo de alzada. Además, la Corte tiene presente, en su considerando cuarto: "Que el artículo 317 expresa que en la cuestión de paternidad -que es la cuestión jurídica de autos-y que interesa para estos efectos es legítimo contradictor 'el padre contra el hijo'". Por lo tanto, la acción de nulidad debió intentarse, aplicando los artículos

Los subrayados son nuestros. 
265 y 317 , en contra de la madre, en su calidad de representante legal del menor, y también en contra del hijo. Es decir, existió, como se reconoce en el considerando sexto de la sentencia, lo que la doctrina denomina litisconsorcio necesario, o sea, aquella situación en la cual una de las partes está compuesta por varios sujetos ${ }^{8}$.

A mayor detalle, precisa el tribunal de alzada en el considerando sexto que: "En el caso de autos se trata de un litisconsorcio necesario impropio al no expresar la ley cuando los varios sujetos deben actuar obligatoriamente en una misma relación procesal, debiendo determinarse en juicio". Este también fue el razonamiento del tribunal de primera instancia, como se hace presente en el considerando quinto: "Así lo entendió además la propia jueza de familia con fecha 5 de noviembre al exigir al actor que la acción debía dirigirse no solo contra el hijo menor sino además en contra de la madre 'parte que habría desplegado la acción dolosa'". La madre fue debidamente notificada, y en dos ocasiones, interrumpiéndose a su respecto la prescripción. Pero, el punto de discusión jurídica se refirió al hijo: establecer si la interrupción de la prescripción que operó respecto de la madre se extiende al menor?.

Como bien observa el sentenciador en el considerando décimo, no existe una norma especial que regule esta materia a propósito del Derecho de familia. Lo contrario sucede en el Derecho de las obligaciones, sede en la que el artículo 2519 del Código Civil establece que, por regla general, la interrupción que obra a favor o en contra de uno de varios coacreedores o codeudores, respectivamente, no aprovecha ni perjudica a los otros sujetos activos o pasivos, salvo que exista solidaridad (lo que debe ser complementado con las obligaciones indivisibles, como segunda excepción, en virtud del tenor del artículo 1529). En este contexto, la Corte decidió acudir a la norma contenida en el artículo 24 del Código Civil, aplicando, en consecuencia, el espíritu general de la legislación y la equidad natural a fin de resolver el conflicto de relevancia jurídica sometido a su decisión.

\section{3.) EL RECURSO DEL SENTENCIADOR AL ESPÍRITU GENERAL DE LA LE- GISLACIÓN Y A LA EQUIDAD NATURAL}

Como ya hemos adelantado, la Corte de Apelaciones de Antofagasta para resolver la contienda analizada, recurrió al artículo 24 del Código Civil, pero en definitiva solo aplicó la equidad y no el espíritu general de la

8 Romero Seguel, Alejandro. (2007). Curso de Derecho Procesal Civil, t. I, Santiago: Editorial Jurídica de Chile, p. 105. Véase, además, Orellana, Fernando. (2010). Manual de Derecho procesal, tomo II, Santiago: Librotecnia, p. 48.

$9 \quad$ En este sentido se pronunció el sentenciador en el considerando noveno: "Que la cuestión que debemos dilucidar, ahora, es si la interrupción producida en el mes de junio de 2009 aprovecha o no al hijo que con posterioridad es demandado". 
legislación. La aplicación del tribunal de alzada de la equidad natural, en nuestro concepto, fue acertada. Si bien es cierto que, en principio, algunos podrían sostener que el Código Civil, para el supuesto de pluralidad de sujetos pasivos, establece como regla general que si se interrumpe la prescripción respecto de uno de ellos, no se entiende interrumpida para el resto (lo que iría en apoyo de la pretensión de los demandados), esta interpretación pasa por alto que el artículo 2519 se refiere a la interrupción de la prescripción respecto de varios sujetos pasivos que se encuentran en una particular situación: han contraído en forma simplemente conjunta una determinada relación. Se trata, entonces, de un supuesto muy preciso de regulación, no directamente aplicable a los juicios de filiación. Tampoco es procedente en este caso emplear la analogía, dado que el mencionado precepto, de claro contenido patrimonial, no guarda relación ni semejanza alguna con la particular naturaleza de los intereses en pugna en los juicios en los que se discute la validez o nulidad del reconocimiento. En efecto, muy distinta es la situación de los deudores que la de los demandados en un juicio en que lo discutido es la existencia de una libre y espontánea voluntad que conduce a la determinación de la paternidad.

En este estado de cosas, la Corte justifica la aplicación del artículo 24 en el considerando décimo: "Que a mayor abundamiento no existe en materia de familia -como si lo existe en materia de derecho patrimonial (artículo 2519 código civil) - una norma que permita precisar si la interrupción aprovecha a los demandados. Por lo anterior debemos a falta de norma expresa recurrir al artículo 24 del Código Civil que ordena interpretar de acuerdo al espíritu general de la legislación y a la equidad natural", es decir, la norma es empleada por el tribunal ad quem como un medio de integración de una laguna jurídica. Pero resulta que del análisis del artículo 24, recurriendo al elemento gramatical de interpretación de la ley, se desprende que dicha norma es un medio de interpretación para aquellos casos en que "no pudieren aplicarse las reglas de interpretación precedentes", respecto de los pasajes "obscuros o contradictorios". Nada se dice de su empleo en el proceso de integración, en principio, se trata de un "medio supletorio de interpretación" 10 , mas esta conclusión no logra explicar completamente la función del artículo 24. En este sentido, en la práctica, se ha utilizado el artículo 24 como precepto que contribuye a la integración de la ley. Por lo tanto, el artículo 24 del Código Civil puede cumplir una doble función: interpretativa e integradora, dependiendo, según el caso, de lo difícil que sea desentrañar el real sentido y alcance de una norma de Derecho positivo o ante la ausencia de norma que dirima la contienda. Además, esta interpretación guarda armonía con el no 5 del 
artículo 170 del Código de Procedimiento Civil, por el cual, las sentencias, a falta de ley, deberán enunciar "los principios de equidad con arreglo a los cuales se pronuncia el fallo". En este sentido se ha pronunciado nuestra doctrina ${ }^{11}$.

Entendemos que acierta nuevamente el tribunal de alzada al fallar que debía rechazarse la excepción de prescripción extintiva deducida por la madre del menor. Justificó la Corte su decisión en lo siguiente: la demandada, al notificársele la demanda, en junio de 2009, "hizo suyo y para su hijo la demanda de nulidad del reconocimiento" 12 , y no alegó, pudiendo, la falta de legitimación o la prescripción en la audiencia preparatoria llevada a cabo el 29 de octubre de 2010. Es decir, existió un comportamiento negligente. Lo contrario puede observarse en el actuar del actor. Como ya se ha señalado, en un primer momento notificó la demanda solo a la madre, con posterioridad, en virtud de la decretada nulidad de todo lo obrado, volvió a notificar a la representante legal del menor, y

11 Para Rodríguez Grez, una vez excluida la analogía, ya sea porque no existe una norma similar o por entenderse esta como obsoleta, el sentenciador debe recurrir, en primer lugar, a los principios generales del Derecho (expresión sinónima, en su opinión, a la de espíritu general de la legislación), y en segundo término, a la equidad, en este orden. Corrobora lo anterior el autor al afirmar que los principios generales del Derecho, que constituyen una síntesis de la legislación en general, son "el más importante y fecundo de los elementos de integración de las lagunas legales (...) Finalmente, si el juez no llegara a encontrar tampoco los principios generales en los cuales afirmar la fórmula de la cual extrae la norma particular destinada a resolver el caso, deberá recurrir a la 'equidad natural'”, Rodríguez Grez, Pablo. (2004). Teoría de la interpretación jurídica. 2a edic., Santiago: Editorial Jurídica de Chile, pp. 27-29 y 81. Ducci ha sostenido que los criterios del art. 24 no son meramente supletorios, sino que, informan todo el proceso interpretativo. Agrega que en un método lógico no puede desconocerse el espíritu general de la legislación y que este forma parte del elemento sistemático. Por su parte, la equidad, hecha copulativa con el espíritu general de la legislación en el precepto legal, debe estar presente cuando se integra o interpreta la ley, de forma de no llegar a soluciones absurdas o inicuas, Ducci. (2006) 151-152. Al parecer la fuente del art. 24 es el art. 21 del Código de Luisiana, así lo entiende Ducci, Ibid., p. 149. Guzmán matiza lo anterior, al decir que es posible que el art. 21 haya servido de inspiración general para la idea de legislar, en forma conjunta con las ideas de Delvincourt, Guzmán Brito, Alejandro. (2007). Las reglas del "Código Civil" de Chile sobre interpretación de las leyes. Santiago: LexisNexis, p. 184. El art. 21 del Código de Luisiana establece lo siguiente "Dans les matières civiles, le juge, à défaut de loi précise, est obligé de procéder conformément à l'équité; pour décidir suivant l'équité, il faut recourir à la loi naturelle et à la raison, ou aux usages reçus, dans le silence de la loi primitive”. El estudio más completo realizado sobre el tema es el de Guzmán, quien explica que el recientemente transcrito art. 21 se inspiró en el artículo 11 del título $5^{\circ}$ del libro preliminar del Project de l'an VIII, Ibid., p. 183. Esta norma prescribía: "Dans les matières civiles, le juge, à défaut de loi précise est un ministre d'équité. La équité est le retour à la loi naturelle, ou aux usages reçus, dans le silence de la loi positive". En los dos últimos preceptos, se aprecia que, en términos expresos, se habla de "equidad" y no de "equidad natural", y además, se consagra a la equidad como un elemento de integración de la ley, lo que no sucede en nuestro Código Civil, y nada se dice del espíritu general de la legislación, esto último, por tanto, sería una innovación de Andrés Bello.

12 Tal fue la opinión del sentenciador expresada en el considerando undécimo. En este considerando se precisa que la demandada en la audiencia preparatoria "dio expresamente su acuerdo a la nulidad de todo lo obrado "a fin de evitar la nulidad que pueda afectar a los actos del proceso y resguardar el interés superior del niño". 
además, al hijo ${ }^{13}$. En apoyo de lo anterior, y en la misma línea de razonamiento, tengamos presente que nadie puede aprovecharse de su propio dolo o negligencia.

Por otra parte, al no reclamar el demandado el vicio procesal que motivó la declaración de nulidad de todo lo obrado, sino que, por el contrario, continuó sus actuaciones procesales, operó la convalidación de dicho vicio, lo que hubiese provocado, seguramente, al continuar el juicio, la dictación de la sentencia, produciéndose, incluso, el saneamiento in limine litis de todos los vicios procesales. Si lo último no ocurrió, fue a causa de la actividad oficiosa del tribunal de primera instancia. Así lo entendió la Corte al establecer en el considerando duodécimo: "Que por lo tanto la equidad ${ }^{14}$ nos lleva a concluir que la madre al ser demandada y además representante legal del menor, al no reclamar legalmente la falta de legitimación convalidó el vicio procesal y por lo tanto no puede con posterioridad aprovecharse de su propia negligencia, y reclamar en el nuevo juicio la prescripción de la acción”. El Fiscal de la Corte de Apelaciones de Antofagasta compartió la opinión de esta, expresando que "la sentencia apelada ha llevado el formalismo a niveles extremos" 15 .

Pero no solamente la equidad permite justificar la resolución de la Corte de Apelaciones. La decisión del tribunal de alzada respeta el espíritu general de la legislación ${ }^{16}$, en concreto, el principio de la protección

Este comportamiento diligente fue reconocido por la Corte de Apelaciones en el considerando decimotercero al sostenerse lo siguiente: "Que sin duda la demanda interpuesta y debidamente notificada el ańo 2009 demuestra por parte del actor la intención real y efectiva de ejercer su derecho a que se declare la nulidad del reconocimiento prestado por él. El demandante ejerció la acción dentro de plazo legal de prescripción, y posteriormente con la declaración de nulidad de todo lo obrado nuevamente - dentro de plazo legal- ejerce real y efectivamente las acciones en contra de los demandados. El actuar del actor ha sido siempre diligente."

14 Sobre la noción de equidad natural, se ha sostenido que esta no es la de capite suo, también denominada cerebrina, es decir, el juez debe seguir la equidad de la ley, no la de su cabeza, en consecuencia, se trata de una categoría objetiva y verificable, la que puede ser argumentada y razonada, "Debemos, pues, evitar pensar que la equidad natural a que alude el artículo 24 del Código sea una puerta abierta a la subjetiva opinión individual, al sentimiento y a la imaginación, que excusa tener que razonar sobre la base de premisas objetivas, ciertas y justificables, probadas por la ciencia y aprobadas por la más autorizada doctrina”, GuzMán. (2007), 203. Además, se ha indicado que la equidad natural es, "es la consideración de un caso particular para darle una solución adecuada a sus exigencias particulares", Ducci. (2006), 156; "un sentido (en cuanto entendimiento o razón o modo particular de entender una cosa o juicio que se hace de ella) intuitivo de la justicia aplicada a un caso o situación concreta, con prescindencia del derecho positivo", RodríGuez Grez. (2004), 86.

15 Opinión contenida en el considerando décimo sexto.

16 Algunos entienden que el espíritu general de la legislación hace referencia a los principios generales del Derecho. En este sentido, Rodríguez Grez. (2004) 84. Quintana, por su parte, afirma que: "La breve explicación que dan los autores sobre la expresión 'espíritu general de la legislación' no permite avanzar demasiado, pero sugiere que se trata de ciertas ideas o directrices que informan la legislación. Esto se aproxima a lo que después se llamarán principios jurídicos" Quintana Bravo, Fernando. (2006). Interpretación y argumentación jurídica. Santiago: Editorial Jurídica de Chile, p. 122. En contra, Guzmán, para quien el espíritu general de la 
de la buena fe, aunque esto no haya sido objeto de pronunciamiento en la sentencia de segunda instancia. Es decir, por exigencias del mencionado principio, el ordenamiento jurídico debe proteger al que está de buena fe, $y$, por el contrario, no otorgar tutela a la mala fe. La mala fe de la representante legal del menor se muestra en querer aprovecharse de un vicio procesal, del cual nunca dijo nada hasta antes de la nulidad de todo lo obrado, para dejar sin efecto la interrupción de la prescripción que le perjudicaba. Insistimos, a sabiendas de que no se había notificado al menor, continuó con sus actuaciones procesales, con lo cual queda claro que no se produjo daño alguno para su representado, para con posterioridad, intentar sacar provecho de la resolución oficiosa del sentenciador.

\section{4) CONCLUSIONES}

En definitiva, consideramos que en el fallo analizado se ha efectuado una adecuada aplicación del Derecho. Especialmente interesante es la interpretación del artículo 24 del Código Civil como elemento de integración de la ley, al aplicar la equidad natural para resolver la litis sometida a su decisión.

Como ya hemos advertido, pensamos que pudo el tribunal, para otorgar mayor fundamento a su resolución, acudir al mismo espíritu general de la legislación, dado que, es razonable pensar, que si un determinado comportamiento es calificado de contrario a la equidad natural, tal conducta puede, además, contradecir el espíritu general de la legislación, en concreto, la protección de la buena fe y la sanción de la mala fe. En caso contrario, habría que concluir que la equidad podría ir en contra del espíritu general de la legislación, afirmación esta última muy difícil de aceptar.

\section{BIBLIOGRAFÍA}

- Abeliuk Manasevich, René. (2003). Las obligaciones, t. II, 4a edic., Santiago (Editorial Jurídica de Chile), 1148 pp.

legislación está referido a la razón o finalidad objetiva no de una ley específica, sino de una cierta generalidad de la legislación, la que puede acotarse por instituciones o figuras, como los derechos reales o las garantías, o por sectores más amplios: el trabajo, el comercio, la minería, etc, GuzMán. (2007) 191-192. Ducci celebra que nuestro legislador no haya utilizado la expresión "principios generales del Derecho, por considerarla de difícil y discutida determinación. Como principios que constituyen el espíritu general de la legislación, menciona el rechazo al enriquecimiento sin causa, la protección de la buena fe, la reserva al Estado de la tutela jurisdiccional de los derechos, la irretroactividad de las leyes, la igualdad de los ciudadanos ante la ley y la libertad individual, Ducci. (2006) 153-155. En definitiva, sin perjuicio de esta disparidad de opiniones, nadie ha sostenido que la protección de la buena fe no forme parte integrante del espíritu general de la legislación. 
- Alessandri R., Arturo, Somarriva U., Manuel y Vodanovic H., Antonio. (2004). Tratado de las obligaciones. Modificación y extinción de las obligaciones, Vol. III, 2a edic., Santiago: Editorial Jurídica de Chile, 269 pp.

- Barcia Lehmann, Rodrigo. (2008). Lecciones de Derecho civil chileno, t. III, Santiago: Editorial Jurídica de Chile, 241 pp.

- Domínguez Águila, Ramón. (2004). La prescripción extintiva. Doctrina y jurisprudencia: Editorial Jurídica de Chile, 435 pp.

- Ducci Claro, Carlos. (2006). Interpretación jurídica, $3^{a}$ edic., Santiago: Editorial Jurídica de Chile, 266 pp.

- Gómez DE LA Torre, Maricruz. (2007). El sistema filiativo chileno. Santiago: Editorial Jurídica de Chile, 519 pp.

- Guzmán Brito, Alejandro. (2007). Las reglas del "Código Civil" de Chile sobre interpretación de las leyes. Santiago: LexisNexis, 258 pp.

- Larraín Ríos, Hernán. (2003). Teoría general de las obligaciones. Santiago: LexisNexis, 600 pp.

- Meza Barros, Ramón. (1994). Manual de Derecho civil. De las obligaciones. Santiago: Editorial Jurídica de Chile, 565 pp.

- Orellana, Fernando. (2010). Manual de Derecho procesal. Tomo II, Santiago: Librotecnia, 412 pp.

- Quintana Bravo, Fernando. (2006). Interpretación y argumentación jurídica. Santiago: Editorial Jurídica de Chile, $338 \mathrm{pp}$.

- Ramos Pazos, René. (2005). Derecho de familia, t II, 5a edic., Santiago: Editorial Jurídica de Chile, 668 pp.

- Rodríguez Grez, Pablo. (2004) Teoría de la interpretación jurídica. $2^{a}$ edic., Santiago: Editorial Jurídica de Chile, $182 \mathrm{pp}$.

- Rodríguez Grez, Pablo. (2008). Extinción no convencional de las obligaciones, Vol. II, Santiago: Editorial Jurídica de Chile, 333 pp.

- Romero Seguel, Alejandro. (2007). Curso de Derecho Procesal Civil, t. I, Santiago: Editorial Jurídica de Chile, 132 pp.

- Troncoso Larronde, Hernán. (2006). De las obligaciones, $4^{a}$ edic., Santiago: LexisNexis, 318 pp.

- Vial Del Río, Víctor. (2003). Manual del Derecho de las obligaciones en el Código Civil chileno. Santiago: Editorial Biblioteca Americana, $356 \mathrm{pp}$. 